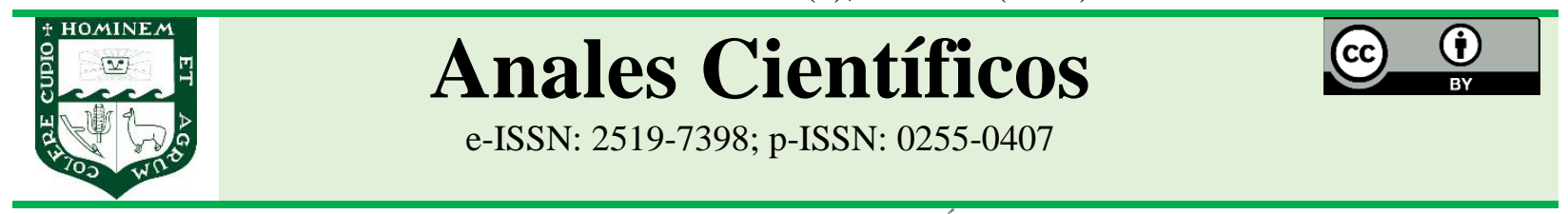

ARTÍCULO ORIGINAL - RESEARCH ARTICLE

http://dx.doi.org/10.21704/ac.v82i2.1785

\title{
INFLUENCIA DEL ACOLCHADO DE PLÁSTICO DE COLOR Y MANTA TÉRMICA EN SANDÍA CV. 'SANTA AMELIA' (Citrullus lanatus Thunb.)
}

\section{Influence of color plastic mulch and thermal blanket in watermelon cv. 'Santa Amelia' (Citrullus lanatus Thunb.)}

\author{
Luis F. Solis-Rosas Diaz ${ }^{1,2 *(D)}$ \\ ${ }^{1}$ Fundo San Felipe, Peruvian Exotics SAC, Perú \\ ${ }^{2}$ Universidad Nacional San Luis Gonzaga de Ica, Ica, Perú. \\ * E-mail: 1fsolisrosas@gmail.com
}

\begin{abstract}
The scope of this study was based on analyzing the productivity, quality and earliness of the watermelon crop cv. 'Santa Amelia' grown on the central coast using polypropylene mulch of different shades using the blanket ends in certain treatments. Eight treatments were studied, mulch white, black and transparent color only, in combination with thermal blanket, thermal blanket alone and control. Completely randomized Block Design was used. The results showed a superior production when using only mulch and mulch more thermal blanket.: Black mulch more thermal blanket with 81,37; white mulch with 75,95; transparent mulch 74,53 and black mulch 74,45 Tn*ha respectively very higher than the control with 45,27 Tn*ha. Regarding the early harvest, the treatment with black mulch in its first harvest achieved 34,75 percent, a result similar to that to which mulch was applied alone and in combination with a thermal blanket, beating the treatment with only thermal blanket with 17,38 and control with 5,1 percent respectively. Regarding the factor of the fruit length over width, being the longest fruit when the factor is higher, the mulch combined with thermal blanket obtained the first place statistically which are the black, white and transparent mulch in combination with thermal blanket with a result of 1,$36 ; 1,34$ and 1,31 respectively.
\end{abstract}

Keywords. Mulch | colors mulch | Citrullus lanatus | thermal blanket | watermelon cultivation

\section{RESUMEN}

El alcance del estudio se basó en analizar la productividad, calidad y precocidad del cultivo de sandía cv. 'Santa Amelia'. El estudio se realizó en la costa central usando acolchado de polipropileno de diferentes tonalidades utilizando la manta térmica en ciertos tratamientos. Se estudió ocho tratamientos, acolchados de color blanco, negro y trasparente, en combinación con manta térmica, manta térmica sola y testigo. Se empleó el Diseño de Bloques Completamente Randomizado. Los resultados mostraron en una producción superior al usarse solamente acolchados y acolchados más manta térmica: Acolchado negro más manta térmica con 81,37; acolchado blanco con 75,95; acolchado trasparente 74,53 y acolchado negro 74,45 $\mathrm{T} *$ ha respectivamente muy superiores al testigo con 45,27 Tn*ha. Con respecto a la precocidad de la cosecha el tratamiento con acolchado negro en su primera cosecha logró el 34,75 \%, resultado similar al a los que se les aplicó acolchado solo y en combinación con manta térmica, 
superando al tratamiento con solo manta térmica con $17,38 \%$ y testigo con $5,1 \%$ respectivamente. Respecto al factor del fruto largo sobre ancho, siendo el fruto más largo cuando el factor es mayor, los acolchados combinados con manta térmica obtuvieron el primer lugar estadísticamente que son el acolchado negro, blanco y trasparente en combinación con manta térmica con resultado de 1,36; 1,34 y 1,31 respectivamente. El uso de acolchado y manta térmica elevó la producción, calidad y concentración de cosecha por la elevación de la temperatura interna del suelo.

Palabras clave: Acolchado | colores acolchado | Citrullus lanatus | manta térmica | cultivo de sandía

Forma de citar el artículo (Formato APA):

Solis-Rosas, L. (2021). Influencia del acolchado de plástico de color y manta térmica en sandía cv. 'Santa Amelia' (Citrullus lanatus Thunb.). 81(2), 227-236. http://dx.doi.org/10.21704/ac.v82i2.1785

Autor de correspondencia (*): Solis-Rosas, L. Email: lfsolisrosas@gmail.com

(C) Los autores. Publicado por la Universidad Nacional Agraria La Molina.

This is an open access article under the CC BY

\section{INTRODUCCIÓN}

El acolchado afecta en el comportamiento hídrico térmico del suelo influyendo en la iluminación, índice de calor, humedad y temperatura en el suelo. Acharya et al. (2005) reportaron que el acolchado se usó para conseguir variar benéficamente el medio ambiente en el suelo. Acelerando la descomposición de materia orgánica (rastrojos de cultivos, entre otros), beneficia en formar una mejor estructura.

El problema para el crecimiento del cultivo de sandía en la zona de Ica es el invierno, porque la temperatura mínima, en la noche es baja, perjudicando a los cultivos en las reacciones metabólicas, bajando la rapidez de las reacciones enzimáticas. Fernández y Johnston, (2006) reportaron que disminuir la temperatura unos pocos grados sobre su umbral adecuado, resulta en un desarrollo poco vigoroso. El producto de la temperatura en el metabolismo define en general el desarrollo del cultivo. Agrolanzarote Servicio Insular Agrario, (2012) reportó que el cultivo de sandía es de clima tropical, debería estar entre 23 y $28{ }^{\circ} \mathrm{C}$ para obtener buen crecimiento. El crecimiento del cultivar de la sandía se ve afectado el desarrollo del cultivo y luego al fruto al ser la planta expuesta a temperaturas menores.

Las disposiciones meteorológicas en la costa central en el departamento y provincia de Ica, respecto a las temperaturas de invierno pueden lograr a ser inferiores a $10{ }^{\circ} \mathrm{C}$, obteniendo como resultado un crecimiento disminuido en el cultivar de la sandía, afectándolo negativamente en rendimiento, calidad y atrasando la cosecha, posicionándolo en una situación complicada para acceder con cosecha temprana y mejores precios. Este experimento trató en estudiar y amortiguar el efecto perjudicial de las bajas temperaturas al iniciar siembra precoz en esta localidad.

El objetivo de la investigación fue estudiar el efecto del emplasticado al suelo "mulch" de diferentes tonalidades (blanco, negro y trasparente) solos y también en combinación con la manta térmica, la cual afecta el calor interno en el suelo relacionado con la concentración e precocidad de cosecha, calidad y producción en esta cucurbitácea (sandía).

\section{MATERIALES Y MÉTODOS}

La investigación se desarrolló en el Fundo "San Felipe" ubicado en la Panamericana sur en el kilómetro 271, en el distrito de salas, provincia y departamento de Ica, Perú. Las fechas de trasplante, fenología y días después de trasplante (DDT) fueron: Trasplante el 08 de agosto con DDT 0, crecimiento acelerado el 04 de setiembre con DDT 27, desarrollo de flores y cuaja el 16 de setiembre con DDT 39, desarrollo del fruto el 06 de octubre con DDT 59, maduración el 15 de octubre con DDT 68, primera cosecha el 27 de octubre con DDT 80, segunda cosecha el 31 de octubre con DDT 84, tercera cosecha el 06 de noviembre con DDT 90, cuarta cosecha el 13 de noviembre con DDT 97 y quinta cosecha el 04 de diciembre con DDT 118. En la tabla 1, se muestran los datos meteorológicos entre enero y diciembre del 2014. 
Tabla 1. Datos meteorológicos del año 2014.

\begin{tabular}{lcccc}
\hline \multirow{2}{*}{ Meses } & \multicolumn{3}{c}{ Temperatura $\left({ }^{\circ} \mathbf{C}\right)$} & Horas de sol mensual \\
\cline { 2 - 4 } & $\begin{array}{c}\text { Temperatura media } \\
\text { máxima mensual }\end{array}$ & $\begin{array}{c}\text { Temperatura media } \\
\text { mínima mensual }\end{array}$ & Temperatura media & 221,2 \\
\hline Enero & 31,0 & 18,1 & 23,1 & 242,2 \\
Febrero & 30,8 & 18,9 & 23,4 & 279,2 \\
Marzo & 32,4 & 20,2 & 25,4 & 248,6 \\
Abril & 30,3 & 17,7 & 24,4 & 156,2 \\
Mayo & 26,5 & 15,3 & 22,3 & 242,8 \\
Junio & 24,6 & 12,4 & 18,4 & 167,2 \\
Julio & 23,5 & 11,5 & 17,5 & 188,6 \\
Agosto & 24,5 & 13,8 & 19,2 & 199,4 \\
Septiembre & 25,3 & 13,7 & 19,7 & 234,5 \\
Octubre & 26,4 & 13,9 & 21,2 & 229,6 \\
Noviembre & 27,4 & 14,7 & 21,2 & 221,1 \\
Diciembre & 28,6 & 16,5 & 23,3 & \\
\hline
\end{tabular}

Fuente: Servicio Nacional de Meteorología e Hidrología del Perú (2014).

Código y descripción de los tratamientos: N: Acolchado negro, B: Acolchado blanco, T: Acolchado trasparente, NM: Acolchado negro en combinación con manta térmica, BM: Acolchado blanco en combinación con manta térmica, TM: Acolchado trasparente en combinación con manta térmica, M: Manta térmica, Tes: Control o testigo.

\section{Diseño experimental}

Se usó el DBCA (Diseño de bloque completamente aleatorizado), con ocho tratamientos y cuatro repeticiones, para la comparación de medias de los tratamientos se usó Duncan (Fernández et al., 2006). Respecto a comparar dos medias de un con acolchado de la misma tonalidad y uno de ellos con manta térmica se empleó la prueba de T. Finalmente se utilizó regresión lineal para determinar si hay un vínculo entre la producción y la temperatura. Se usó el software SAS 8,0 y Microsoft Excel 2010 para obtener los resultados estadísticos.

\section{Instalación de los tratamientos}

Después del acondicionamiento del suelo se instalaron tres cintas de riego presurizado a $20 \mathrm{~cm}$ entre ellas por línea de cultivo, el mulch o acolchado se pusieron sobre el suelo y las cintas de riego, se fijó en los extremos de la cama poniéndole tierra encima a los lados.

Después se procedió a realizar los hoyos para posteriormente trasplantar los plantines. Luego que los plantines se instalaron inmediatamente se puso la manta termina en los tratamientos que les correspondía, luego a los costados de la manta térmica se le colocó tierra para evitar que se mueva con el viento.

Cabe acotar que la manta termina se puso simplemente encima de los platines, ya que como es muy ligera la planta al desarrollarse no tiene problemas en alzarla. Se instaló una colmena a 10 metros de la zona experimental 5 días antes de extraer la manta termina la cual se quitó apenas se inició la floración femenina para que estas sean visitadas por las abejas (polinización entre la flor macho con la hembra).

\section{Análisis físico-químico del agua para riego:} pH 9,92; C.E 3,56; Calcio 22;53 meq/l; magnesio 4,32 meq/l; potasio $0,31 \mathrm{meq} / 1$; sodio $9,31 \mathrm{meq} / \mathrm{l}$; nitratos $2,9 \mathrm{meq} / \mathrm{l}$; alcalinidad $\left(\mathrm{CO}_{3} \mathrm{H}^{-}\right)$0,93 meq/l; sulfatos $8,51 \mathrm{meq} / \mathrm{l}$; cloruros $23,82 \mathrm{meq} / \mathrm{l}$; boro $0,19 \mathrm{ppm}$; hierro <0,05 ppm; manganeso <0,05 ppm; zinc <0,05 meq/l; cobre < 0,05 ppm; RAS 2,54; dureza 1345. Según la clasificación en salinidad y sodicidad está en C4 - S1. Tiene un nivel de dureza alto resultando en 1345 ppm. Además, los cloruros están en nivel elevado.

\section{Análisis físico-químico del suelo \\ Textura:}

Arena 90,22\%; arcilla 6,00\%; Limo 3,78\%.

\section{Clase textura: Arenoso}

En cuanto a la saturación Porcentaje está en 24,2\%; carbonato de calcio 1,15\%; conductividad eléctrica (extracto de saturación) 7,97 dS/m; pH 7,92; fosforo 
disponible 4,58 ppm; materia orgánica $<0,03 \%$; nitrógeno total $<0,0017 \%$; potasio disponible 115,54 ppm.

\section{Cationes cambiables:}

Calcio 1,54 meq/100; magnesio 0,59 meq/100; sodio 0,59 meq/100; potasio 0,28 meq/100; PSI 19,67\%; CIC 3.00

Sales solubles:

Cloruro 50,42 meq/100; sulfato 30,86 meq/100; nitrato 2,91 meq/100; carbonato $0,00 \mathrm{meq} / 100$; bicarbonato 1,8 meq/100; calcio 45,05 meq/100; magnesio 8,76; sodio 31,43 meq/100; potasio 0,49 meq/100; Boro 2,20 ppm.

\section{Especificaciones de herramientas e insumos:}

El material vegetal usado fue del cultivar de sandía "Santa Amelia" (hibrido).

El mulch o acolchado es de material de polietileno de alta densidad, su color es solamente blanco, negro y trasparente, el ancho de este fue de 2,0 m.
La manta térmica es de polipropileno poroso, muy ligera y fuerte al rompimiento, tuvo como ancho $2,5 \mathrm{~m}$.

\section{RESULTADOS Y DISCUSIÓN}

\section{Temperatura interna del suelo en grados centígrados $\left({ }^{\circ} \mathrm{C}\right)$}

En la Tabla 2, se presenta la temperatura media interna del suelo media en el periodo que abarca puesta la manta termina y después sin esta. Cuando la manta termina estaba puesta el TM con $29,17{ }^{\circ} \mathrm{C}$ fue el ganador estadísticamente, del segundo hasta el quinto lugar fueron T, NM, BM y N que resultaron en 27,77; 27,32; 26,74 y $25,94{ }^{\circ} \mathrm{C}$ respectivamente, como sexta posición estuvieron $\mathrm{M}$ y B con resultados 24,58 y 24,56 ${ }^{\circ} \mathrm{C}$ respectivamente, en última posición lo obtuvo el TES resultando en $22,34^{\circ} \mathrm{C}$.

Cuando se extrajo la manta termina el ganador fue el T obteniendo $27,22^{\circ} \mathrm{C}$, como segunda posición quedó el TM obteniendo $26,85^{\circ} \mathrm{C}$, NM y $\mathrm{N}$ obteniendo 25,83 y $25,78^{\circ} \mathrm{C}$ respectivamente obtuvieron el tercer lugar la aplicación de B y BM obteniendo 25,22 y $25,14{ }^{\circ} \mathrm{C}$ respectivamente ocuparon la cuarta posición, finalmente la última posición fue del B y BM con 25,22 y $25,14{ }^{\circ} \mathrm{C}$ respectivamente.

Tabla 2. Temperatura promedio en el interior de suelo con la manta térmica y sin esta, Longitud del brote principal y Cantidad de frutos por planta por cada tratamiento

\begin{tabular}{|c|c|c|c|c|c|c|c|c|}
\hline \multirow[t]{2}{*}{ Tipo tratamiento } & \multicolumn{4}{|c|}{$\begin{array}{c}\text { Temperatura promedio en el interior del } \\
\text { suelo }\end{array}$} & \multicolumn{2}{|c|}{$\begin{array}{l}\text { Longitud del } \\
\text { brote principal } \\
\text { (m) }\end{array}$} & \multicolumn{2}{|c|}{$\begin{array}{l}\text { Cantidad de frutos } \\
\text { por cada planta } \\
\text { (und) }\end{array}$} \\
\hline & \multicolumn{2}{|c|}{$\begin{array}{l}\text { Incluye la manta } \\
\text { térmica }\end{array}$} & \multicolumn{2}{|c|}{$\begin{array}{l}\text { Al quitar Manta } \\
\text { termina manta térmica }\end{array}$} & & & & \\
\hline & Días de 0 a 3 & & Días de & 8 a 73 & & & & \\
\hline $\mathrm{N}$ & 25,94 & $\mathrm{e}$ & 25,78 & $\mathrm{c}$ & 4,20 & $\mathrm{a}$ & 2,82 & $a^{*}$ \\
\hline B & 24,56 & $\mathrm{f}$ & 25,22 & $\mathrm{~d}$ & 3,78 & $\mathrm{~b}$ & 2,52 & $\mathrm{~b}$ \\
\hline $\mathrm{T}$ & 27,77 & $\mathrm{~b}$ & 27,22 & $\mathrm{a}$ & 4,02 & $\mathrm{a}$ & 2,41 & $\mathrm{~b}$ \\
\hline NM & 27,32 & $\mathrm{c}$ & 25,83 & $\mathrm{c}$ & 3,68 & $\mathrm{~b}$ & 2,57 & $\mathrm{~b}$ \\
\hline BM & 26,74 & $\mathrm{~d}$ & 25,14 & $\mathrm{~d}$ & 3,73 & $\mathrm{~b}$ & 2,23 & $\mathrm{c}$ \\
\hline TM & 29,17 & a & 26,85 & $\mathrm{~b}$ & 3,67 & $\mathrm{~b}$ & 2,53 & $\mathrm{~b}$ \\
\hline M & 24,58 & $\mathrm{f}$ & 22,96 & $\mathrm{e}$ & 3,52 & $\mathrm{c}$ & 2,11 & $\mathrm{c}$ \\
\hline TES & 22,34 & $\mathrm{~g}$ & 23,00 & $\mathrm{e}$ & 3,66 & $\mathrm{~b}$ & 1,88 & $\mathrm{~d}$ \\
\hline C.V (\%) & 1,03 & & 0,47 & & 6,87 & & 4,97 & \\
\hline Promedio & 26,05 & & 25,25 & & 3,78 & & 2,38 & \\
\hline
\end{tabular}

Cabe resaltar que el color del acolchado influye cuando en el periodo con manta térmica para algunos tratamientos como el TM con $29,17{ }^{\circ} \mathrm{C}$ y $\mathrm{T}$ con 27,77
${ }^{\circ} \mathrm{C}$, ocuparon el primer y segundo lugar. $\mathrm{Y}$ en el periodo sin manta termina para todos los tratamientos nuevamente el tratamiento T ocupó el primer lugar con 
27,22 ${ }^{\circ} \mathrm{C}$. En ambos casos el TES quedó en último lugar. La eficacia del acolchado de plástico trasparente en aumentar la temperatura se debe a que este permite el ingreso de los rayos solares y afecta directamente al suelo además guarda el calor. Los plásticos de color negro y blanco simplemente estos se calientan y transfieren el calor al suelo por contacto además de guardar calor, esta data y análisis son parecidos a lo experimentado por Acharya et al., (2005) en el cual manifiestan que el uso de plástico en el suelo o mulch afecta el estado hídrico térmico en el suelo.

Como data adicional el tratamiento $\mathrm{T}$ si no se le aplica herbicida pre emergentes puedes tener problemas malezas ya que estas siguen recibiendo luz solar, como lo recalcaron Yaghi et al., (2013) que al aplicar acolchado que deja pasar la luz aumenta más la temperatura del suelo y el plástico de color oscuro tiene como ventaja o inhibición de malezas.

\section{Longitud de brote en metros (m)}

La Tabla 2, se refiere al largo del brote principal de los experimentos $\mathrm{N}$ y $\mathrm{T}$ con 4,20 y 4,02 tuvieron como posición el primer lugar, seguido de B, BM, NM, TM y TES con 3,$78 ; 3,73 ; 3,68 ; 3,67$ y $3,66 \mathrm{~m}$ respectivamente, finalmente, el $\mathrm{M}$ con $3,52 \mathrm{~m}$ se posicionó como último lugar estadísticamente.

La aplicación de la manta no tuvo relación positiva en la longitud del brote, tal vez porque al retenerlo inicialmente dentro de ella lo limita o tenga nuevos brotes el cual le hacen competencia posterior a la guía o brote principal.

\section{Análisis de la cantidad de frutos por cada planta (und)}

En la Tabla 2, se observa que en la variable cantidad de frutos por planta en unidades, $\mathrm{N}$ obtuvo 2,82 frutos se posicionó como ganador, en segunda posición estuvieron NM, TM, B y T, con 2,57; 2,53; 2,52 y 2,41, la tercera posición lo ocuparon BM y M con 2,23 y 2,11 respectivamente y en última posición TES obteniendo 1,88 frutos.

Otra apreciación es que los acolchados incluyendo manta térmica incrementaron la cantidad de frutos por cada planta como comentaron Wilson et al., (1995) que usando acolchados aumentan estadísticamente la cantidad de frutos por cada planta y además también obedecían a una calidad óptima para su comercialización.

Para obtener un número de frutos importante por planta es necesario tener la masa foliar adecuada para mantenerlos, por lo tanto, la temperatura debe ser ideal para su desarrollo y buen cuaje de frutos. El frío del invierno con una media mínima mensual de $13,8{ }^{\circ} \mathrm{C}$ en agosto se mitigó con el uso de mulch o acolchado cubriendo el suelo y manta de polipropileno en sus respectivos tratamientos.

Tabla 3. Comparación de 2 medias con prueba de $\mathrm{T}$ de acolchado del mismo color con manta y sin manta.

\begin{tabular}{|c|c|c|c|}
\hline \multicolumn{4}{|l|}{ Prueba de $\mathrm{T}$} \\
\hline Hipótesis nula vs Hipótesis alternativa & $\begin{array}{l}\text { Estadístico de } \\
\text { prueba }\end{array}$ & T tabla & $\begin{array}{l}\text { Criterio } \\
\text { decidir }\end{array}$ \\
\hline$N M-N=0$ vs $N M-N>0$ & 7,26 & & \\
\hline $\mathrm{BM}-\mathrm{B}=0$ vs $\mathrm{BM}-\mathrm{B}>0$ & 11,46 & & \\
\hline $\mathrm{TM}-\mathrm{T}=0$ vs $\mathrm{TM}-\mathrm{T}>0$ & 7,40 & 1.72 & tc > t $(0,95 ; 21)$ \\
\hline$M-$ TES $=0$ vs $M-$ TES $>0$ & 11,8 & & \\
\hline
\end{tabular}

\section{Influencia de la temperatura interna en el suelo respecto al empleó de manta térmica}

En la Tabla 3, se comparan por pares que coinciden en la tonalidad del mulch su única diferencia es que uno tuvo manta termina y el otro no, se formaron cuatro grupos usando la prueba de $\mathrm{T}$. Todos resultaron mayores a 1.72 ( $\mathrm{T}$ tabla a la confianza de $95 \%$ y grados de libertad 21). Se puede afirmar estadísticamente que en todos los grupos del mismo color de acolchado los tratamientos que tuvieron manta térmica fueron 
superiores en temperatura cuando esta manta estuvo puesta, incluso el tratamiento $\mathrm{M}$ resultó significativamente superior al TES, resultado similar a lo comentado por Ortiz et al., (2010) al emplear manta termina de polipropileno esta mantiene la temperatura y humedad, favoreciendo al óptimo desarrollo del cultivo. Por lo tanto, se acepta en todas las comparaciones la hipótesis alternativa.

\section{Producción de fruta en toneladas por hectárea (Tn} $\mathbf{h a}^{-1}$ )

En la Tabla 4, tenemos que los tratamientos que posicionaron como ganadores fueron NM, B, TM y N obteniendo 81,37; 75,95; 74,53 y 74,45 Tn ha ${ }^{-1}$ respectivamente, en segunda posición $\mathrm{T}$ obteniendo 69,72 $\mathrm{Tn} \mathrm{ha}^{-1}$, tercera posición BM obteniendo 63,30 Tn ha ${ }^{-1} \mathrm{y}$ última posición $\mathrm{M}$ y TES con 51,20 y 45,27 Tn ha-1.

Tabla 4. Producción de fruta $\left(\mathrm{Tn} \mathrm{ha}^{-1}\right)$.

\begin{tabular}{lc}
\hline Tipo tratamiento & Producción Tn ha ${ }^{-1}$ \\
\hline $\mathrm{N}$ & $74,45 \mathrm{a}^{*}$ \\
$\mathrm{~B}$ & $75,95 \mathrm{a}$ \\
$\mathrm{T}$ & $69,72 \mathrm{~b}$ \\
$\mathrm{NM}$ & $81,37 \mathrm{a}$ \\
$\mathrm{BM}$ & $63,3 \mathrm{c}$ \\
$\mathrm{TM}$ & $74,53 \mathrm{a}$ \\
$\mathrm{M}$ & $51,2 \mathrm{~d}$ \\
$\mathrm{TES}$ & $45,27 \mathrm{~d}$ \\
\hline C.V. $(\%)$ & 8.6 \\
Promedio: & 66,97 \\
*Letras diferentes indicas diferencias significativas, prueba \\
Duncan (p<=0,05). \\
C.V. $(\%):$ Coeficiente de variación en porcentaje.
\end{tabular}

Debido a que el cultivo de sandía requiere temperaturas mayores a la registradas en su mes de trasplante que fue el 8 de agosto con $13,8{ }^{\circ} \mathrm{C}$ de media mínima mensual para su desarrollo optimo, la utilización de acolchados y manta térmica obtuvieron una producción muy superior al testigo. El aumento de rendimiento fue por el efecto de acumulación de calo en el suelo y este irradia calor hacia la planta ya que esta es rastrera. Este comportamiento al usar esta tecnología es similar a lo que reportaron Cenobio et al., (2006) que en el cultivar de sandía en los tratamientos que se empleó acolchado aumentaron la temperatura significativamente en el suelo, teniendo como resultado un desarrollo más vigoroso, veloz y por ende se aumentó el rendimiento.

El incremento de rendimiento también se da por mayor eficacia de absorber nutrientes porque al subir la temperatura llegando a la óptima del cultivo esta tiene un metabolismo ideal y por ende mejora la absorción de minerales nutritivos de la solución suelo.

Cabe recalcar que Earhart et al., (1991) reportaron que el cultivo de sandía la extracción de nutrientes aumentó en nitrógeno 18 , fosforo 16 , calcio 22 y magnesio $15 \%$. Siempre y cuando se use acolchado.

Ibarra et al. (2006) reportaron que en el cultivo de sandía el uso de acolchados es adecuado para generar un mayor rendimiento.

\section{Análisis de la concentración de cosecha en porcentaje $(\%)$.}

La figura 1, muestra concentración de cosecha en porcentaje en base al rendimiento total en su primera cosecha hizo acumularla para después. En la primera cosecha todos los tratamientos con acolchado y en combinación con manta termina ocuparon la primera posición siendo el mejor tratamiento con acolchado negro con $34,75 \%$ siendo más precoces que el $\mathrm{M}$ con 17,38 y TES con $5,1 \%$.

En la segunda cosecha ocuparon la primera posición en concentración de cosecha los tratamientos NM con 40,40; B con 36,38; TM con 36,20 y T con 32,35\%. En segunda posición se tiene al BM con 28,33 y M con $26,03 \%$. Sin embargo, el tratamiento que obtuvo una gran concentración de cosecha fue el $\mathrm{N}$ obteniendo la última posición con $9,93 \%$ debido a su volumen cosechado anteriormente.

En la tercera cosecha el tratamiento Tes en concentración de cosecha ocupó nuevamente el primer lugar al igual que la segunda cosecha con 33,05\%, entonces se puede afirmar que el retraso. 


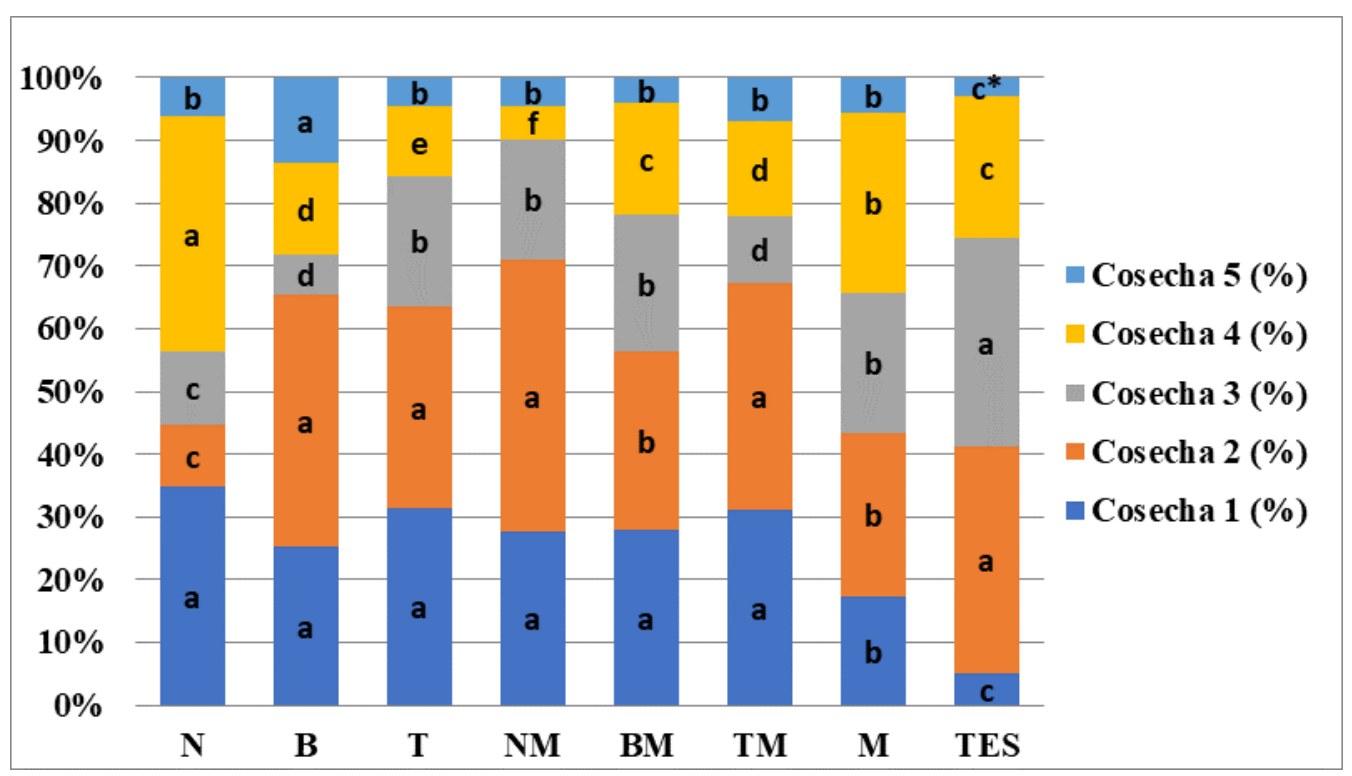

Figura 1. Concentración de cosecha en porcentaje en base al rendimiento total por cosecha. *Letras diferentes indicas diferencias significativas, prueba Duncan $(\mathrm{p}<=0,05$

En la cuarta cosecha el tratamiento $\mathrm{N}$ ocupa el primer lugar al igual que su primera cosecha con $37,48 \%$, en segunda posición está el tratamiento M con $28,55 \%$, en tercera posición se encuentra TES con 22,83 y BM con $19,95 \%$, en cuarta posición está el TM con 15,10 y B obteniendo $14,50 \%$, en quinta posición está $\mathrm{T}$ resultando en $11,08 \%$ y última posición está el NM $5,35 \%$.

En la última cosecha siendo la quinta, el tratamiento B ocupó la primera posición con $5,35 \%$, en segunda posición quedó TM 6,93; N con 6,00; M 5,68; T con 4,$68 ; \mathrm{NM}$ con 4,43 y BM con $3,98 \%$ y tercer y última posición quedó el TES con 2,83\%.

En los tratamientos que se usó acolchado de plástico de diferentes colores y también los que tuvieron acolchado hicieron el cultivo más precoz en dar su primera cosecha en concentración, resultado similar a Cenobio et al., (2006) en el cual investigaron el uso de acolchados de plástico en sandía, tuvo como resultado un aumento en la temperatura del suelo, por ende desarrollo optimo y más acelerado del cultivo en llegar a la cosecha, se obtuvo entre 9 a 11 días de adelanto en comparación a los tratamiento que no tuvieron acolchado.
Tener en cuenta que en la tercera cosecha el tratamiento que más acumulo cosecha respecto al $100 \%$ fue el NM que llegó al 90,18\%. A pesar que el tratamiento TES ocupó los primeros lugares en orden de mérito en la segunda y tercera cosecha esto no significa que obtuvo un rendimiento ideal. Lo expuesto también coincide con Hernández et al., (2011) quienes reportaron que en el hibrido "Santa Amelia" a los tratamientos que se le aplicó acolchado negro la cosecha tuvo una precocidad de 30 días.

Respecto a la sanidad del cultivo según Ortiz et al., (2010) reportaron que cuando se aplica manta termina al cultivo este funciona como una barrera para evitar que ingresen los áfidos y evita la propagación de virus por estos vectores. Obteniendo el $100 \%$ del potencial productivo aun cuando la manta térmica se extraiga para su polinización.

También Brown et al., (1993) reportaron que el acolchado los colores de plástico blanco, amarillo y negro fueron intermedios sobre la población de áfidos y la reducción de la enfermedad ocasionada por virus, el plástico reflectante retraso de 10 a 13 días los síntomas de la enfermedad. 


\section{Afinidad temperaturas del suelo con la producción}

Se aplicó un análisis de regresión lineal para saber la relación entre la temperatura del suelo y la producción del cultivo de sandía. En la Figura 2, se puede concluir que hay relación directa entre la temperatura y el rendimiento. Por cada grado centígrado que se añade en el interior del suelo la producción se eleva en 5,54 toneladas por hectárea.

\section{Peso promedio por fruto}

En la Tabla 5, los tratamientos que ocuparon la primera posición son NM con 9,50; B con 9,08; y TM con 8,85 $\mathrm{kg}$, en segunda posición T con 8,68 y BM con $8,53 \mathrm{~kg}$, en tercera posición $\mathrm{N}$ con $7,9 \mathrm{~kg}$ y el cuarto o último lugar $\mathrm{M}$ con 8,28 y TES con 7,2 kg por fruto.

Se observa que la aplicación de manta térmica y acolchado obtuvieron los pesos por fruto más grandes en comparación a los que solo se utilizó manta y testigo.

El uso de acolchado eleva la producción de esta cucurbitácea (sandía) estando acorde con Acharya et al., (2005) quienes reportaron que mejora el rendimiento de los cultivos.

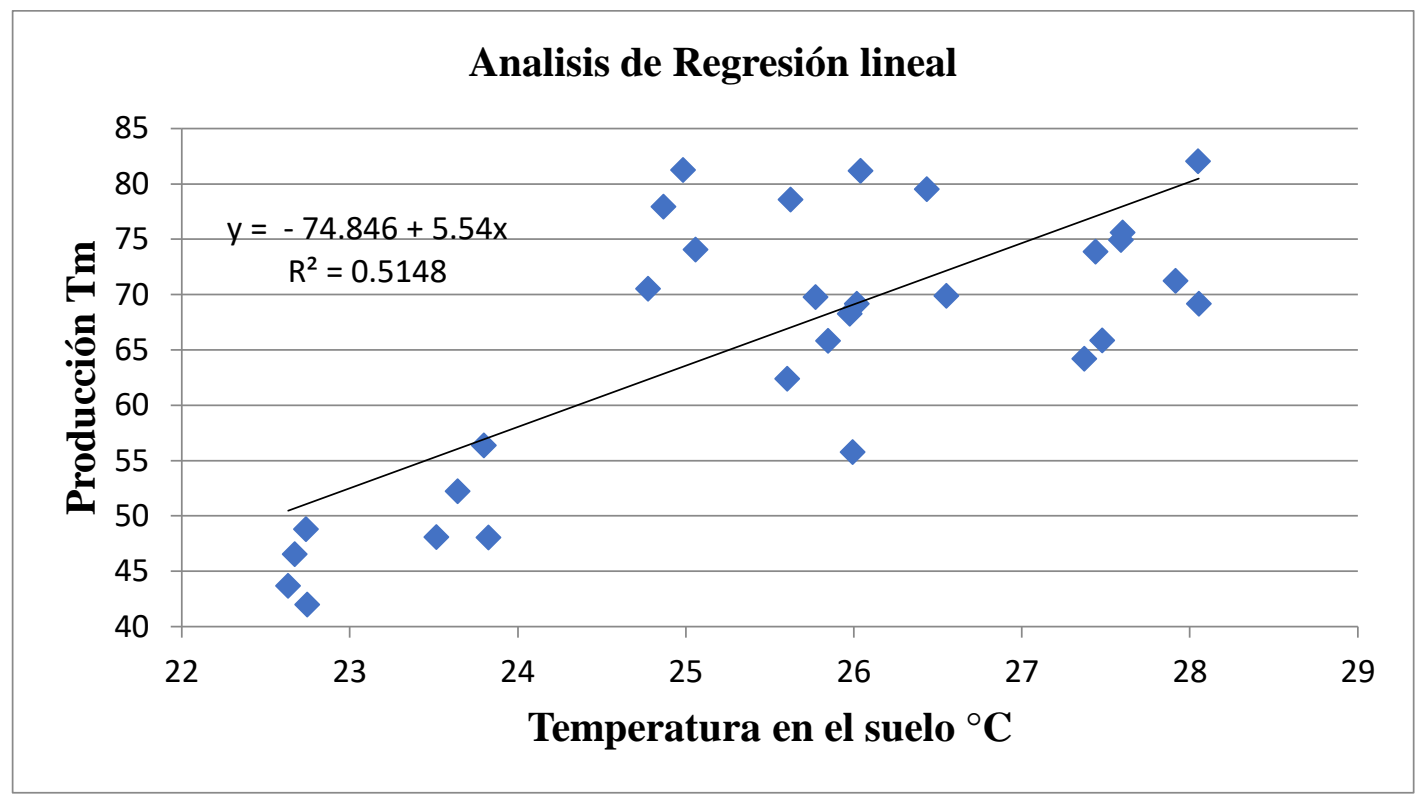

Figura 2. Afinidad entre la temperatura del suelo ${ }^{\circ} \mathrm{C}$ y la producción.

Tabla 5. Peso por fruto promedio en $\mathrm{kg}$, longitud y ancho del fruto en $\mathrm{cm}$, relación largo sobre el ancho y grados Brix en frutos del cultivo de sandía.

\begin{tabular}{|c|c|c|c|c|c|c|c|c|}
\hline \multirow[t]{2}{*}{ Tipo tratamientos } & \multirow{2}{*}{$\begin{array}{l}\text { Peso por fruto } \\
\text { promedio en } \\
\text { kg }\end{array}$} & \multicolumn{3}{|l|}{ Tamaño } & \multirow[t]{2}{*}{$\begin{array}{l}\text { Relación } \\
\text { sobre ancho }\end{array}$} & \multirow[t]{2}{*}{ largo } & \multirow{2}{*}{\multicolumn{2}{|c|}{ Grados Brix }} \\
\hline & & Longitud & Ancho & & & & & \\
\hline $\mathrm{N}$ & $7,90 \quad \mathrm{c}$ & $30,67 \quad b$ & 24,25 & $\mathrm{a}$ & 1,27 & & 11,83 & $\mathrm{c}^{*}$ \\
\hline B & 9,08 a & 31,36 a & 24,61 & $\mathrm{a}$ & 1,28 & & 12,00 & $\mathrm{~b}$ \\
\hline $\mathrm{T}$ & $8,68 \quad b$ & 31,03 a & 24,14 & $\mathrm{a}$ & 1,29 & & 11,38 & $\mathrm{~d}$ \\
\hline NM & 9,50 a & 32,53 a & 24,05 & $\mathrm{a}$ & 1,36 & & 12,50 & $\mathrm{a}$ \\
\hline BM & $8,53 \mathrm{~b}$ & 31,61 a & 23,67 & $\mathrm{a}$ & 1,34 & & 11,25 & $\mathrm{e}$ \\
\hline $\mathrm{TM}$ & 8,85 a & 31,52 a & 24,03 & $\mathrm{a}$ & 1,31 & & 12,18 & $\mathrm{a}$ \\
\hline M & $7,28 \mathrm{~d}$ & $29,39 \mathrm{c}$ & 23,15 & $\mathrm{~b}$ & 1,27 & & 10,5 & $\mathrm{f}$ \\
\hline TES & $7,20 \mathrm{~d}$ & $30,28 \quad b$ & 24,11 & $\mathrm{a}$ & 1,26 & & 12,68 & $\mathrm{a}$ \\
\hline C.V (\%) & 5,49 & 3,56 & 2,48 & & 2,36 & & 2,98 & \\
\hline Promedio & 8,38 & 31,05 & 24,00 & & 1,29 & & 11,79 & \\
\hline
\end{tabular}

*Letras diferentes indicas diferencias significativas, prueba Duncan $(\mathrm{p}<=0,05)$.

C.V. (\%): Coeficiente de variación en porcentaje. 


\section{Longitud y ancho por fruto}

En la tabla 5, se aprecia el tamaño y forma del fruto con la data largo, ancho y la división de estas. A medida que la relación largo sobre ancho aumenta, el fruto es más largo.

Los tratamientos de mayor largo de fruto NM con 32,53; BM con 31,61; TM con 31.52; B con 31,36; y T con $31,03 \mathrm{~cm}$, en segunda posición $\mathrm{N}$ con 30,67; y TES con $30,28 \mathrm{~cm}$, en tercera y última posición está el M con $29,39 \mathrm{~cm}$.

En el ancho del fruto ocuparon la primera posición B con 24,61; $\mathrm{N}$ con 24,25; $\mathrm{T}$ con 24,14; TES con 24,11; $\mathrm{NM}$ con 24,05; TM con 24,03 y BM con 23,67 cm y en última posición el único tratamiento fue el $\mathrm{M}$ con $23,15 \mathrm{~cm}$.

En la relación longitud sobre el ancho del fruto ocuparon la primera posición los tratamientos NM con 1,36 ; BM con 1,34 y TM con 1,31, ocuparon el segundo o última posición T con 1,29; B con 1,28; M con 1,$27 ; \mathrm{N}$ con 1,27 y TES $1,26 \mathrm{~cm}$.

Los tratamientos NM, BM y TM obtuvieron data similar posicionándose primeros en jerarquía tanto en largo y ancho del fruto, aparte estos mismos tratamientos tienen un factor resultante de la división largo sobre ancho más alta teniendo un fruto más alargado y grande. Al aumentar la temperatura inducidos por mulch y manta inciden en la calidad de la fruta respecto a su forma incidiendo en una mejor cuaja. Estos resultados coinciden con Ortiz et al., (2010) quienes reportaron que al usar manta térmica esta crea un micro clima favorable para el cultivo, teniendo un incremento de calidad y producción además de ser eficiente en el control de áfidos.

\section{Análisis de los ${ }^{\circ} B r i x$ de la pulpa del fruto de sandía} En la Tabla 5, se obtuvo el ${ }^{\circ}$ Brix del medio de la pulpa de cada fruto, siendo los ganadores el TES con 12,68; $\mathrm{NM}$ con 12,50 y $\mathrm{TM}$ con $12,18{ }^{\circ}$ Brix, en segunda posición está el B con $12,00^{\circ}$ Brix, en tercera posición está el $\mathrm{N}$ con $11,83^{\circ} \mathrm{Brix}$, en cuarta posición está el tratamiento $\mathrm{T}$ con $11,38^{\circ} \mathrm{Brix}$, en quinta posición está el BM con $11,25^{\circ}$ Brix y en sexta y última posición está el M con $10,5^{\circ}$ Brix.
Cabe recalcar que el TES está en primera posición junto al NM y TM, el tratamiento TES a pesar de su bajo rendimiento y calidad, es posible que haya acumulado más grados brix por el menor número de frutos que tenía por planta ya que derivó más fotosintatos a una menor cantidad de fruta.

\section{CONCLUSIONES}

En conclusión, la variedad de sandía sandía cv. 'Santa Amelia' (Citrullus lanatus Thunb.) es susceptible al frio. Mientras que, la aplicación de la tecnología con acolchado y manta térmica eleva la producción, concentración de cosecha, longitud y cantidad de frutos por planta debido a la elevación de la temperatura interna del suelo por el efecto del acolchado. El acolchado transparente presenta temperatura superior con y sin manta térmica. Se recomienda realizar estudios en otras variedades.

\section{Conflictos de intereses}

Los autores firmantes del presente trabajo de investigación declaran no tener ningún potencial conflicto de interés personal o económico con otras personas $\mathrm{u}$ organizaciones que puedan influir indebidamente con el presente manuscrito.

\section{Contribuciones de los autores}

Preparación y ejecución: LSR; Desarrollo de la metodología: LSR; Concepción y diseño: LSR; Edición del artículo: LSR; Supervisión del estudio: LSR.

\section{REFERENCIAS}

- Acharya, C. L.; Hati, K. M., \& Bandyopadhyay, K. K. (2005). Encyclopedia of soils in the environment.

https://www.sciencedirect.com/science/article/pii/ B0123485304002502

- Agrolanzarote servicio insular agrario. (2012). Fichas técnicas de cultivos de Lanzarote. http://www.agrolanzarote.com/sites/default/files/ Agrolanzarote/02Productos/documentos/agrolanza rote.ficha_calabacin.pdf

- Brown, J. E.; Dangler, J. M.; Woods, F. M.; Tilt, K. M.; Henshaw, M. D.; Griffey, W. A., \& West, M. 
S. (1993). Delay in Mosaic Virus Onset and Aphid Vector Reduction in Summer Squash Grown on Reflective Mulches. HortScience, 28(9), 895-896.

- Cenobio, G.; Inzunza, M. A.; Mendoza, S. F.; Sánchez, I., \& Román, A. (2006). Acolchado plástico de color en sandía con riego por goteo. Terra Latinoamericana, 24(4), 515-520.

- Earhart, D. R.; Dainello, F. J., \& Baker, M. L. (1991). Yield responses and nutrient uptake of triploid watermelon as affected by fertilizer source, mulch, and row cover. HortScience, 26(5), 484498.

- Fernández, G.; Johnston, M. (2006). Crecimiento y temperatura. Universidad de la Serena.http://exa.unne.edu.ar/biologia/fisiologia.v egetal/Crecimientoytemperatura.pdf

- Fernández R., Trapero A., \& Dominguez, J. (2010). Experimentación en Agricultura. Consejería de Agricultura y Pesca. Servicio de Publicaciones. Sevilla.

- Hernández, J.; Medina, R., \& Hernández, O. (2011). Evaluación del híbrido de sandía Santa
Amelia (Citrullus lanatus Thunb.) en tres tipos de cobertura. Agronomía colimbiana, 19(2), 54-66.

- Ibarra, L.; Quezada, R.; Cedeño, B.; Lozano, A. J., \& De la Rosa, M. (2006). Watermelon Response to Plastic Mulch and Row Covers. European journal of horticultural science, 71(6), 262-266.

- Ortiz, M.; Medina, R.; Valdivia, R.; Ortiz, A.; Alvarado, S., \& Rodríguez, J. R. (2010). Mosquitas blancas plaga primaria de hortalizas en Nayarit. Revista fuente, 5, 31-40.

- Servicio Nacional de Meteorología e Hidrología del Perú. (2014). Datos meteorológicos Estación Villacuri, Ica.

- Wilson, M.; Khan, V., \& Stevens, C. (1995). Influence of several nondegradable mulches and one biodegradable mulch on yield of Crimson Sweet Watermelon. HortScience, 30(3), 426-444.

- Yaghi, T., Arslan, A., \& Naoum, F. (2013). Cucumber (Cucumis sativus L.) water use efficiency (EUA) under plastic mulch and drip irrigation. Agricultural water management, 128, 149-157. 